Version of Record: https://www.sciencedirect.com/science/article/pii/S0009898119301123

LC-MS/MS measurements of urinary guanidinoacetic acid and creatine: method optimization by deleting derivatization step

Rucheton Benoit ${ }^{1, *}$ benoit.rucheton@aphp.fr, MesliSamir ${ }^{2}$, Julian Boutin ${ }^{2}$, Amintas Samuel ${ }^{2}$, Colombies Brigitte ${ }^{2}$, Ducint Dominique ${ }^{2}$, Redonnet -Vernhet Isabelle $e^{2,3}$

${ }^{1}$ Metabolic Biochemistry Department, Pitié Sal pêtrière University Hospital, AP-HP, Paris, France

${ }^{2}$ Clinical Chemistry Department, Inherited Metabolic Diseases, CHU Bordeaux, 33076 Bordeaux, France

${ }^{3}$ INSERM U 1211: MRGM Rare diseases, genetic and metabolism, Bordeaux University, 33000 Bordeaux, France

*Corresponding author at: Hôpitaux Universitaires Pitié-Salpêtrière - Charles Foix 47/83 boulevard de I'Hôpital 75013 PARIS

\title{
Abstract
}

Background: Cerebral Creatine deficiency syndromes (CCDS) include three hereditary diseases affecting the metabolism of creatine $(\mathrm{Cr})$ : arginine glycine amidinotransferase deficiency, guanidinoacetate methyltransferase deficiency and disorders of creatine transporter. These pathologies cause a brain creatine deficiency responsible of non-specific neurological impairments with mental retardation. LC-MS/MS measurements of guanidinoacetic acid (GAA) and creatine in urine and plasma are an important screening test to identify the deficit. Analysis of this polar and basic molecules not hold on standard column requires a derivatization step to butyl-esters. To overcome this long and fastidious derivatization, an ion pairing (IP) method was chosen in this study. Method: IP method was validated using Comité francais d'accréditation (COFRAC) recommendations. Then, urine GAA and creatine of 15 patients with a CDS deficiency suspected were tested y LCMS/MS using IP technique, and performances were assessed with reference laboratory method (butylation method). Moreover, references values were suggested $y$ the study of 100 urines samples of healthy patients.

Results: The method developed provided a good accuracy and precision with intra and inter-day coefficients of variation (CVs) less than $15 \%$. The curve was linear for the biological and pathological concentrations. The comparison with the reference method did not reveal any significant difference for analytical performances but showed a simplification of the preparation of samples. 
Conclusion: The use of IP technique that we have developed demonstrated a good correlation with the butylation method. Moreover, this new method not only allows a simplification of the technique, but also decreases in run time.

Keywords: Cerebral Creatine deficiency syndromes, Mass spectrometry, Guanidinoacetic acid, Creatine, Ion pairing

Abbreviations

CCDS Cerebral Creatine deficiency syndromes

$\mathrm{Cr}$ creatine

GAA guanidinoacetic acid

COFRAC Comité francais d'accréditation

CV coefficient of variation

ATP adenosine triphosphate

AGAT arginine: glycine amidinotransferase

GAMT guanidinoacetate methyltransferase

CRTR or SLC6A8 a Sodium / Chlorine-dependent transmembrane transporter

CNS central nervous system

BBB blood brain barrier

H-MRS magnetic resonance proton spectrometry

CSF cerebrospinal fluid

LC-MS/MS

ACN Acetonitrile

FA Formic acid

MRM multiple-reaction monitoring

Introduction

Creatine allows intracellular energy storage by a mechanism of phosphate exchange with adenosine triphosphate (ATP) mainly in tissues with high energy needed like muscle fibers ( $95 \%$ of creatine contained in human body), and brain [1]. A balanced diet (mainly meat and fish) supplies half of the creatine [2]. The other half comes from three amino acids (glycine, arginine and methionine) by endogenous synthesis.

Creatine synthesis requires two enzymes: L- arginine: glycine amidinotransferase (AGAT, EC 2.1.4.1) and $\mathrm{N}$ - guanidinoacetate methyltransferase (GAMT, EC 2.1.1.2) (fig1). The first step, catalyzed by AGAT mostly expressed in kidney, is the transfer of the amino residue of arginine to glycine and leads to the production of ornithine and guanidinoacetic acid (GAA)[1-3]. The second step is the methylation of the GAA catalyzed by GAMT in liver [1-3]. Creatine, endogenous or ingested, reach 
target tissues from the bloodstream via an active mechanism requiring a X-linked creatine transporter: CRTR or SLC6A8, a Sodium / Chlorine-dependent transmembrane transporter. Intracellular creatine, mostly found in muscle, is reversibly converted, by creatine kinases, into phosphocreatine, a high energy compound used to regenerate ATP. About $1.7 \%$ of total creatine (creatine-phosphocreatine) is converted into creatinine per day by a non-enzymatic mechanism (spontaneously dehydrating and cyclization) [4]. Creatinine then passively diffuses out of the cell and is eliminated in urine. Creatine is an energy reserve molecule for different organs but also seems to play a role in central nervous system (CNS) on neuroprotection, as an antioxidant [4] and on GABA neurotransmission [4-5]. CNS expresses AGAT, GAMT and CRTR [6-7]. CRTR is highly expressed at the blood brain barrier (BBB) during the first years of life but its expression decreases with brain maturation [6-7]. In addition, there is a dissociation of the expressions of AGAT and GAMT in the different types of CNS cells [6-7]. Less than half of CNS cells express both enzymatic activities. Cell co-operations with cell exchanges by CRTR are required for creatine synthesis [8].

Anomalies of one of these molecules (AGAT, GAMT or CRTR) are responsible of inborn errors of creatine metabolism known under the designation of Creatine deficiency syndromes (CCDS). CCDS prevalence is unknown, probablyunderestimated, but evaluated at $2.7 \%$ of the mental retardation [9]. Transmission and treatment depends on the deficit, but all primary creatine disorders result in an absence of creatine in the CNS. CCDS typically lead to a global development delay, intellectual delay and neurological impairment as epilepsy and extrapyramidal involvements [7-10].

Nowadays, the only treatment is based on oral supplementation with creatine monohydrate. Clinical benefits have been shown in patients with GAMT or AGAT deficiency: improvement of non-verbal (visual perception, fine movements) and verbal skills (spontaneous speech, appropriate use of a coherent and intelligible language), improvement of socio-emotional behavior and mood control [1011]. These effects were associated with an increase in cerebral creatine during patient follow -up by proton spectroscopy [11]. In GAMT deficiency, in addition to creatine supplementation, to limit the production of neurotoxic GAA, an ornithine supplementation associated with a dietary arginine restriction is used [12].

For CRTR disorders, supplementation seems to be ineffective and supplementation with precursor amino acids (L-arginine and L-glycine) was tested with varied and controversial results [13].

When CCDS is suspected, GAA, creatine and creatinine are reliable biochemical markers. They can be supplemented by the brain magnetic resonance proton spectrometry (H-MRS): a non-invasive neuroimaging technique that allows an in-vivo diagnosis [11].

The determination of these metabolites in plasma, urine and cerebrospinal fluid (CSF) makes it possible to diagnose the disease and to define the deficit : GAA, GAMT or CRTR [14-15]. GAA and 
creatine are polar and basic molecules. Measurement of such molecules, not held on conventional columns, requires usually an esterification step with butanol. This method, although reliable and with an analytical performance provided, is long and laborious due to the derivatization step [16]. To get round this problem we used ion pairing method. It consists in addition, in the two mobile phases, of an ion of opposite charge to the molecules to be assayed (fig2-a), which together form an uncharged complex. The opposite ion generally have a hydrophobic chain which, by interaction with the stationary organic phase, will allow a greater retention of the complex and therefore of the molecule of interest (fig2-b) [17-18]. Different molecules can be used for ion pairing method such as perfluoropentanoic acid.

The purpose of this work is to develop and validate a new LC-MS/MS method for the measurement of creatine and guanidinoacetate by eliminating the derivatization step and secondly, to compare it with the reference method.

Materials and methods:

Chemicals

Acetonitrile (ACN), Formic acid (FA) were from Biosolve-chemicals. Creatine, GAA and perfluoropentanoic acid were purchased from Sigma Aldrich. It was chosen to use isotopes of creatine and GAA: d2-GAA and d3-creatine (CDN Isotopes, Quebec) as internal standards (IS). Quality controls (QC) SKML (MCA laboratory) and External quality assurance (ERNDIM) were used to validate the method.

Patient specimen collection

CCDS suspected urine samples were sought, collected and then centriguged $10 \mathrm{~min}$ at $3500 \mathrm{G}$. The supernatants were kept frozen at $-80^{\circ} \mathrm{C}$ until analysis.

Standards, IS and quality controls

The standard solutions are made in water by diluting GAA and creatine stock solutions. The final concentrations are showed in the table 1.

An aqueous solution of IS is produced by mixing stock solutions for a final concentration of 150 $\mu \mathrm{mol} / \mathrm{L}$ of d2-GAA and $1000 \mu \mathrm{mol} / \mathrm{L}$ of d3-creatine (Table 1 ). 
Three control solutions were prepared at three different concentrations: $1-\mathrm{GAA}: 90 \mu \mathrm{mol} / \mathrm{L}$, creatine: $320 \mu \mathrm{mol} / \mathrm{L}, 2-\mathrm{GAA}: 170 \mu \mathrm{mol} / \mathrm{L}$, creatine : $3000 \mu \mathrm{mol} / \mathrm{L}, 3-\mathrm{GAA}: 1300 \mu \mathrm{mol} / \mathrm{L}$, creatine : $9000 \mu \mathrm{mol} / \mathrm{L}$ by another person that prepared the calibration points in order to eliminate a bias in preparation.

Sample preparation lon pairing method

Standard solutions, QC and patient's sample urines were diluted to 1/20th in a solution of internal standard (Water/ $0.1 \% \mathrm{AF}$ ) in a 96 well plate. After agitation, they have been analyzed by LC-MS/MS (fig3-a)

\section{Reference method}

Samples were prepared according to the procedure described by Van Noolen et al [18]:50 $\mu \mathrm{L}$ of the $1 / 10$ diluted sample (urine, control or range) are placed in a hemolysis tube with $50 \mu \mathrm{L}$ of IS solutions, then were evaporated to dryness under a nitrogen stream/flux. After a complete evaporation, $300 \mu \mathrm{L}$ of butanol $/ \mathrm{HCl} 3 \mathrm{~mol} / \mathrm{L}$ are added to each tube and were kept $15 \mathrm{~min}$ in a $65^{\circ} \mathrm{C}$ water bath. A second evaporation step to dryness under a nitrogen stream/flux eliminates butanol. Dried samples were resuspended in $200 \mu \mathrm{l}$ of a $0.1 \%$ formic acid water mixture and then transferred into vials or plates to be injected into the LC system (fig3-b).

\section{HPLC and mass spectrometry}

For both method, chromatographic separation was carried out using an ALLIANCE 2795 HT -WATERS ${ }^{\circledR}$ system and the compounds (GAA, creatine) were separated by injecting $5 \mu \mathrm{L}$ of the sample on a Xterra ${ }^{\circledR} \mathrm{C} 18$ column (particle size $3.5 \mu \mathrm{m}, 2.1 \times 30 \mathrm{~mm}$ ) provided by Waters (Guyancourt, France). It was decided to work with a flow rate of $300 \mu \mathrm{L} / \mathrm{min}$ with an elution gradient starting at $100 \%$ of phase $A$ (water/ $0.1 \%$ FA) to $7 \mathrm{~min}$, followed by 2 min of gradient using $30 \%$ of phase $A$ and $70 \%$ of phase $B$, followed by a a wash period of 1 min using $100 \%$ of phase $A$. For the ion pairing technique, $200 \mu$ l of PFPA was added to 1 liter of the two phases.

GAA and Creatine detection and quantification was performed by a first-generation tandem mass spectrometer system QUATTRO MICRO ${ }^{\circledR}$ (Waters) with an electrospray ionization source. Datas were acquired by MRM (multiple-reaction monitoring) mode and conditions for mass spectrometry analysis are summarized in the table 2 .

\section{Quantification}

Concentrations were determined using a linear regression calibration curve of the ratio of area of compound to the area of the IS. This ratio eliminates variations in extraction yields, injection volumes 
or variations in the source. Datas are acquired via the Masslyn $x^{\circledR}$ software which area under curve ratio into directly usable results: GAA or creatine concentrations.

\section{Method validation}

To validate this method, the COFRAC (comité français d'accréditation) recommendations has been used as reference and adapted to the specific criteria of chromatographic dosages. Homemade QC, SKML QC were used for the study of intra and inter run precisions of this method. ERNDIM QC were chosen to ensure accuracies. Homemade $Q C$ and the first standard range point are analyzed 5 times a day for 3 successive days. For each of them, the mean concentration, coefficient of variation and bias: (100x (C target-C target) / target C) are calculated. An Anova test is performed for each level of controls to determine: repeatability: intra-day CV, reproducibility: inter-day CV. For long-term performance evaluation of this method, SKML CQs were measured on 10 different assay runs. Inaccuracies were determined by comparing values obtained of ERNDIM QC with the target values and by estimating biases and z-scores. The limit of quantification (LOQ) is determined experimentally by performing 5 assays for low concentrations and corresponds to the lowest point for which repeatability, reproducibility and bias are $<20 \%$ (not data show). Three ranges were carried out to study linearity correlation coefficient and the bias between the theoretical and measured concentration of the range points were compared. Matrix effect was measured by comparing peak's areas obtained with and without matrix (water buffer). It was tested with 6 different urines that have been analyzed in triplicate for each urine) (not data shown).

\section{Method Comparison}

15 urine samples from patients with a CCDS deficiency suspected were tested for GAA and creatine. Analytical performances were assessed using a Passing-Bablock regression analysis to define the Pearson correlation coefficient and the Bland-Altman mean difference plot.

References values

Urine samples from 100 patients, 37 girls and 63 boys were analyzed to determine normal values of GAA and creatine rates. Results were compared with the reference values realized in French population [19] with the butanol esterification method. Usual values are contained between the 2, 5 and the 97,5 percentiles.

Results

Validation method 
GAA and the d2-GAA were eluted at $2.67 \mathrm{~min}$, creatine and d3-Creatine at $3.1 \mathrm{~min}$. The range of calibration was chosen to cover the biological and pathological values. For both, GAA and creatine, calibration curves were linear between 0-1500 $\mu \mathrm{mol} / \mathrm{l}$ and 0-10000 $\mu \mathrm{M}$ respectively. The mean of the coefficient of regression were 0.999 for GAA and creatine. For all the range points, the bias between theoretical and measured concentration was less than $15 \%$ on the calibration curves

The intra-run precision obtained with homemade QC was good for GAA and creatine (CV $<5 \%$ for both). The inter-run precision CV was less than $6 \%$ fo GAA and creatine (table 3). All were lower than $15 \%$ for the two molecules which proves the fidelity of our method.

Accuracy was estimated by external quality assurance samples, 14 EQ (ERNDIM) were good with biases lower than $15 \%$ and with z-score lower than 2 (table 4). For long-term monitoring of the performance of our method, the coefficients of variation for the two levels of SKML CQs were 8.2\% and $5.3 \%$ for GAA (fig $4-\mathrm{a}$ ) and $2.1 \%$ and $3.7 \%$ for the creatine (fig4-b). There is a very large matrix effect of about $150 \%$ for GAA and $172 \%$ for creatine that varies according to the urine but is the same for the IS. The use of the area ratio of the peak of the molecule on the area of the peak of it IS makes it possible to compensate for this matrix effect.

\section{Method comparison}

15 urine samples were analyzed with both methods. The student test showed no significant difference between the concentrations of GAA and creatine obtained by the two methods $(p=0.25$ for GAA and $p=0.77$ for creatine) (fig5-a). A BLAND-ALTMAN graph compares the average of the measurements with their corresponding percentages of differences ([butylation method] - [lon Pairing method]) / average. Most of the differences are within the range given by the approval limits $d$ (mean of percentage of difference) \pm 2 sdd (standard deviation of percentage of difference). Only one point for GAA was higher than 2sdd but very close. $(d=15.72,2 s d d=15.54)$ (fig5-b). The Passing-Bablock regressions show strong correlations between the results of the two methods for the two molecules (GAA $r=0.991$, creatine $r=0.99$ ) (fig5-c). Confidence intervals $(\mathrm{Cl})$ for intercepts include 0 that there is no systematic difference between the two methods (GAA -18.2 to 15.2, creatine -56.4 to 70.9$)$. Cl for slopes include 1 , there is no proportional difference between the two techniques (GAA 0.869 to 1.049 , creatine 0.852 to 1.071 ) (table 5).

Usual values 
Hundred suspected CCDS urines were analyzed. Only patients with normal values, compared to the French paper/study were included in this estimation. Results are standardized by urinary creatinine levels to correct fluctuations related to diuresis ( $\mu \mathrm{mol} / \mathrm{mol}$ creatinine). Values (all ages considered) obtained for boys were: 16.32 to $116 \mu \mathrm{mol} / \mathrm{mol}$ creatinine for GAA (mean: $57 \pm 33$ ) and 20.27 to $1131 \mu \mathrm{mol} / \mathrm{mol}$ creatinine for creatine (mean =368 \pm 328 ) and for girls 15.18 to $163.3 \mu \mathrm{mol} / \mathrm{mol}$ creatinine for GAA (mean : $77 \pm 40.3$ ) and 24.28 to $1098 \mu \mathrm{mol} / \mathrm{mol}$ creatinine for creatine (mean $=407.7 \pm 350.1)$. These results are very similar to those described previously in a French population by Joncquel-Chevalier Curt et al. [19]

\section{Discussion:}

The new method developed for the determination of GAA and creatine is linear on concentrations which range from physiological to pathological values. Method validation showed a good precision and accuracy with intra- and inter-day CVs and mean bias less than $15 \%$. Registration for the External Quality Assurance Program ERNDIm QC allowed us to compare this technique with all assay methods of these two molecules. It confirmed a good accuracy without biases obtained. These good results enabled us to implement this method in our laboratory, and an analysis of the two SKML CQIs passed to each series of assays showed correct CVs (2.7 to $8.2 \%)$ confirming the results of the initial validation and the preservation of this accuracy over the time. The comparative analysis of GAA and creatine assays for 15 urines by the two methods proved that there was no significant difference in the values obtained by our method from those obtained by the reference method. The Bland-Altman concordance analysis shows that there is a positive bias ( 3.29 for GAA, 2.87 for creatine) but not significant ( 0 being within the confidence interval: $G A A=-11.95$ to 18.52 , creatine $=-15.03$ to 21.06 ). Bland-Altman diagram indicates only one sample with a difference between the concentrations of GAA measured by both methods outside of acceptable limits but very closed. The Passing and Bablok regression suggest a good correlation between the two methods (GAA: $r=0.991$; Creatine $r=0.990$ ). The study of slope and intercept at the origin does not reveal a systematic proportional difference (the confidence intervals of the slopes include the 1 and those of the intercept at the origin comprise the 0 ). It should be noted that for GAA the concordance between the two techniques is less good for concentrations $<100 \mu \mathrm{mol} / \mathrm{L}$ according to the Passing-Bablock curve. This comparative study is confirmed by the good results achieved in the external quality con trol program since most of the laboratories enrolled in this program use the butylation method. Moreover, the estimation of the reference values by the new technique in our laboratory has found values very similar to those described in the French population obtained by the old method. Both methods therefore seem to have similar performance. The implementation of this technique in our laboratory made it possible to diagnose two abnormalities of the CRTR transporter which were confirmed by 
molecular biology. It should be noted that there is a significant matrix effect on the urine samples (no data shows). This is compensated by the use of deuterated IS.

The sample preparation time was also compared and long stages as evaporation were suppressed. Indeed the IP method requires only one dilution whereas the butylation method needs two complete evaporation steps and a 15 minutes water bath. Sample were prepared in $30 \mathrm{~min}$ for IP technique against about $2 \mathrm{~h}$ for the butylation one. That greatly simplified the method and decreased the total time of the sample preparation by 0.75 from 2 hours to 30 minutes. Removal of the butylation step made it possible to no longer use concentrated acid and thus to avoid the corrosive action of the latter on the evaporators. Moreover, the absence of evaporation highlighted of the possibility of producing larger series because the number placed on the evaporator was usually limited. It is then easier to carry out studies incorporating these assays, to estimate its own normal values and incorporate this analysis into all investigations prescribed in the context of mental retardation or resistant epilepsy. The CDS is a group of pathologies with an under-estimated frequency and sometimes reversible mental retardation under treatment. The interest of this rapid and effective technique would increase the number of cases diagnosed and allow a treatment and thus a correction of mental retardation more effective.

\section{Conclusion}

The IS method developed for the determination of GAA and creatine is a more simple and efficient technique than the Butylation method. The validation and comparison study revealed good fidelity, good accuracy and performances comparable to the reference method. The use of the pairing ion corresponds to an optimization that makes it possible to reduce the number of manual steps, using corrosives products, and thus to increase the frequency of the assays series. The implementation of this technique in our laboratory showed a good persistence of the fidelity on the CQI and we carried out the diagnosis two CCDS for two patients. All results show that our method is efficient for the diagnosis of CCDS and comparable to the previous technique.

\section{References}

[1] Joncquel-Chevalier Curt M, Voicu PM, Fontaine M, Dessein AF, Porchet N, Mention-Mulliez K, et al. Creatine biosynthesis and transport in health and disease. Biochimie. 2015;119:146-65. 
[2] J.T. Brosnan, M.E. Brosnan, Creatine: endogenous metabolite, dietary, and therapeutic supplement, Annu. Rev. Nutr. 27 (2007) 241-261.

[3] Fons C, Campistol J, Creatine Defects and Central Nervous System, Semin Pediat Neurol. 2016, 23:285-289.

[4] M. Wyss, A. Schulze, Health implications of creatine: can oral creatine supplementation protect against neurological and atherosclerotic disease, Neuroscience 112 (2) (2002) $243 e 260$.

[5] Hanna-El-Daher L., Braissant O. Creatine synthesis and exchanges between brain cells: what can be learned from human creatine deficiencies and various experimental models, Amino Acids. 2016

[6]. Braissant O. Creatine and guanidinoacetate transport at blood-brain and blood-cerebrospinal fluid barriers. J. Inherit. Metab. Dis. 2012;35:655-664

[7] C. Stromberger, O.A. Bodamer, S. Stockler-Ipsiroglu, Clinical characteristics and diagnostic clues in inborn errors of creatine metabolism, J. Inherit. Metab. Dis. 26 (2e3) (2003) 299e308.

[8] Y. Koga, H. Takahashi, D. Oikawa, T. Tachibana, D.M. Denbow, M. Furuse, Brain creatine functions to attenuate acute stress responses through GABAnergic system in chicks, Neur oscience 132 (1) (2005) $65 \mathrm{e} 71$

[9] Lion-François L., Cheillan D., Pitelet G., Acquaviva-Bourdain C., Bussy G., Cotton F., Guibaud L., Gérard D., Rivier C., Vianey-Saban C., et al. High frequency of creatine deficiency syndromes in patients with unexplained mental retardation. Neurology. 2006;67:1713-1714.

[10] V. Leuzzi, Inborn errors of creatine metabolism and epilepsy: clinical features, diagnosis, and treatment, J. Child Neurol. 17(Suppl. 3) (2002) 3589.

[11] Rackayova V, Cudalbu C, Pouwels PJW, Braissant O. Creatine in the central nervous system: From magnetic resonance spectroscopy to creatine deficiencies. Anal Biochem. 2017 Jul 15;529:144-157

[12] Stockler-Ipsiroglu S., van Karnebeek C., Longo N., Korenke G.C., Mercimek-Mahmutoglu S., Marquart I., Barshop B., Grolik C., Schlune A., Angle B., et al. Guanidinoacetate methyltransferase (GAMT) deficiency: Outcomes in 48 individuals and recommendations for diagnosis, treatment and monitoring. Mol. Genet. Metab. 2014;111:16-25.

[13] Jaggumantri S, Dunbar M, Edgar V, Mignone C, Newlove T, Elango R, Collet JP, Sargent M, Stockler-Ipsiroglu S, van Karnebeek CD, Treatment of Creatine Transporter (SLC6A8) Deficiency With Oral S-Adenosyl Methionine as Adjunct to L-arginine, Glycine, and Creatine Supplements, Pediatr Neurol. 2015 Oct;53(4):360-363.e2.

[14] N.M. Verhoeven, G.S. Salomons, C. Jakobs, Laboratory diagnosis of defects of creatine biosynthesis and transport, Clin. Chim. Acta 361 (2005) 1. 
[15] Sharer JD, Bodamer O, Longo N, Tortorelli S, 5, Wamelink MM, Young S. Laboratory diagnosis of creatine deficiency syndromes: a technical standard and guideline of the American College of Medical Genetics and Genomics. Genet Med. 2017 Feb;19(2).

[16] Van Noolen L, Monneret D, Ducros V, Corne C, Lunardi J, Faure P. Simultaneous determination of guanidinoacetate, creatine and creatinine by liquid chromatography-tandem mass spectrometry: a diagnostic tool for creatine deficiency syndromes in body fluids and a perspective use on cultured fibroblasts, Ann Biol Clin , 2013 Jul-Aug; 71(4):429-37

[17] T. Cecchi, lon-pairing chromatography, Crit. Rev. Anal. Chem. 38 (2008) 161-213.

[18] F. Michopoulos, N. Whalley, G. Theodoridis, I.D. Wilson, T.P. Dunkley,S.E. Critchlow, Targeted profiling of polar intracellular metabolites using ion-pairhigh performance liquid chromatography and -ultra high performance liquid chromatography coupled to tandem mass spectrometry: applications to serum, urine and tissue extracts, J. Chromatogr. A 349 (2014) 60-68.

[19] Joncquel-Chevalier Curt M, Cheillan D, Briand G, Salomons GS, Mention-Mulliez K, Dobbelaere D, Cuisset JM, Lion-François L, Des Portes V, Chabli A, Valayannopoulos V, Benoist JF, Pinard JM, Simard G, Douay O, Deiva K, Tardieu M, Afenjar A, Héron D, Rivier F, Chabrol B, Prieur F, Cartault F, Pitelet G, Goldenberg A, Bekri S, Gerard M, Delorme R, Porchet N, Vianey-Saban C, Vamecq J, Creatine and guanidinoacetate reference values in a French population. Mol Genet Metab. 2013 Nov;110(3): 263-7 
Table 1 : Calibration points. The range of calibration was chosen to frame the biological and pathological values

\begin{tabular}{|c|c|c|c|c|c|c|c|}
\hline Standard & 1 & 2 & 3 & 4 & 5 & 6 & 7 \\
\hline GAA concentration $(\mu \mathrm{mol} / \mathrm{L})$ & 15 & 75 & 150 & 375 & 750 & 1125 & 1500 \\
\hline Creatine concentration $(\mu \mathrm{mol} / \mathrm{L})$ & 100 & 500 & 1000 & 2500 & 5000 & 7500 & 10000 \\
\hline
\end{tabular}

Table 2 : Instrument parameters for the mass spectrometry analysis of the IP method using the multiple reaction monitoring mode

\begin{tabular}{|c|c|c|c|c|}
\hline Parameters & GAA & d2-GAA & Creatine & d3-creatine \\
\hline Ionisation mode & ESI+ & ESI+ & ESI+ & ESI+ \\
\hline Capillary voltage (V) & 2 & 2 & 2 & 2 \\
\hline Cone voltage (V) & 20 & 20 & 20 & 20 \\
\hline Extractor voltage (V) & 2 & 2 & 2 & 2 \\
\hline Source temperature $\left({ }^{\circ} \mathrm{C}\right)$ & 125 & 125 & 125 & 125 \\
\hline Desolvatation temperature $\left({ }^{\circ} \mathrm{C}\right)$ & 400 & 400 & 400 & 400 \\
\hline Cone gas flow $(\mathrm{l} / \mathrm{h})$ & 50 & 50 & 50 & 50 \\
\hline Desolvatation gas flow $(\mathrm{l} / \mathrm{h})$ & 700 & 700 & 700 & 700 \\
\hline Collision energy (eV) & 15 & 10 & 15 & 15 \\
\hline Precusor ion & 118 & 120 & 132 & 135 \\
\hline Fragment ion & 76 & 78 & 90 & 93 \\
\hline
\end{tabular}

Table 3 : Intra- and interday accuracy and fidelity for GAA and creatine home made Quality Controls.

\begin{tabular}{l|l|l|l|l|}
\cline { 2 - 5 } & LOQ & QC1 & QC2 & QC3 \\
\hline
\end{tabular}




\begin{tabular}{|c|c|c|c|c|c|}
\hline \multirow{4}{*}{ GAA } & mean ( $\mu \mathrm{mol} / \mathrm{L})$ & 15 & 90 & 570 & 1300 \\
\cline { 2 - 6 } & Bias (\%) & -10.09 & -2.84 & -1.04 & -3.03 \\
\cline { 2 - 6 } & CV intra-day (\%) & 7,9 & 4,9 & 3,8 & 2,8 \\
\cline { 2 - 6 } & CV inter-day (\%) & 14 & 4,9 & 5,7 & 2,8 \\
\hline \multirow{5}{*}{ Creatine } & mean ( $\mu \mathrm{mol} / \mathrm{L})$ & 100 & 320 & 3000 & 9000 \\
\cline { 2 - 6 } & Bias (\%) & 2.73 & -1.03 & 3.64 & -2.47 \\
\cline { 2 - 6 } & CV intra-day (\%) & 6,6 & 3,6 & 4 & 3,1 \\
\cline { 2 - 6 } & CV inter-day (\%) & 6,9 & 5 & 5,2 & 4,6 \\
\hline
\end{tabular}

Table 4 : Precision established by the External Quality Assessment EQA analysis -ERNDIM

\begin{tabular}{|c|c|c|c|c|c|c|c|c|}
\hline & \multicolumn{9}{|c}{ GAA } & \multicolumn{4}{c|}{ creatine } \\
\hline EQA & mean all Iab & results & biaises & z-score & mean all Iab & results & biaises & z-score \\
\hline 1 & 23,8 & 23 & $-3,36$ & 0,4 & 7648 & 7366 & $-3,69$ & 0,5 \\
\hline 2 & 27 & 26,4 & $-2,22$ & 0,2 & 7580 & 8100 & 6,86 & 1 \\
\hline 3 & 188 & 186 & $-1,06$ & 0,2 & 3481 & 3400 & $-2,33$ & 0,2 \\
\hline 4 & 484 & 475 & $-1,86$ & 0,2 & 71 & 70 & $-1,41$ & 0,1 \\
\hline 5 & 965 & 945 & $-2,07$ & 0,2 & 898 & 830 & $-7,57$ & 1,1 \\
\hline 6 & 990 & 1075 & 8,59 & 1 & 922 & 850 & $-7,81$ & 1,1 \\
\hline 7 & 203 & 190 & $-6,40$ & 0,8 & 3584 & 3400 & $-5,13$ & 0,8 \\
\hline 8 & 38 & 38 & 0,00 & 0,1 & 7894 & 8366 & 5,98 & 0,9 \\
\hline 9 & 992 & 1070 & 7,86 & 0,9 & 918 & 930 & 1,31 & 0,1 \\
\hline 10 & 200 & 208 & 4,00 & 0,3 & 904 & 842 & $-6,86$ & 0,4 \\
\hline 11 & 998 & 1050 & 5,21 & 0,7 & 7994 & 8300 & 3,83 & 0,4 \\
\hline 12 & 501 & 477 & $-4,79$ & 0,6 & 3595 & 3550 & $-1,25$ & 0,2 \\
\hline 13 & 34 & 38 & 11,76 & 0,6 & 58 & 50 & $-13,79$ & 0,2 \\
\hline 14 & 194 & 211 & 8,76 & 1,1 & 900 & 820 & $-8,89$ & 0,9 \\
\hline
\end{tabular}

Table 5 : Summary of the results of the comparative analysis of the concentrations of GAA and creatine obtained by the IP method and the old method,

\begin{tabular}{|c|c|c|}
\hline & GAA & Creatine \\
\hline \multicolumn{3}{|l|}{ Passing Bablock } \\
\hline Slope (IC 95\% ) & $0,972(0,869$ to 1,049$)$ & $0,985(0,852$ to 1,071$)$ \\
\hline Intercepts (IC 95\%) & $-2,2(-18,2$ to 15,1$)$ & $-0,6(-56,4$ to 70,9$)$ \\
\hline $\mathrm{R}$ & 0,991 & 0,990 \\
\hline \multicolumn{3}{|l|}{ Bland-Altman } \\
\hline Biais (IC 95\%) & $3,29(-11,95$ to 18,52$)$ & $2,87(-15,03$ to 21,06$)$ \\
\hline \multicolumn{3}{|l|}{ Student } \\
\hline$p$ & 0,25 & 0,77 \\
\hline
\end{tabular}

Figure 1 : Metabolism of creatine. One part of creatine is synthetized from 3 amino acids: Arginine, Glycine and Methionine. Two enzymes are required for its synthesis: AGAT (L-arginine-glycine amidinotransferase) and GAMT (guanidinoacetate méthyltransferase)

The first step results in the formation of guanidinoacetate which is methylated by adenosyl methionine to give creatine. Once synthesized, creatine penetrates in muscles via a transporter CRTR. Then it is transformed in phosphocreatine by 
ATP in order to be an energy reserve. Creatine and Phosphocreatine can be converted in creatinine by spontaneous cyclisation which be eliminated in urine.

Figure 2 : Principle of lon Pairing. The principle is to add an opposite charging ion to the molecules of interest in the two mobile phases to form an uncharged complex (A): for example Perfluoropentanoic acid. The opposite charging ion, perfluoropentanoic acid, will interact via its hydrophobic chain with the organic stationary phase causing a stronger retention of the complex.

Figure 3 : Sample preparation . A : IP method. Samples are diluted in a IS (internal standard) solution and vortexed during $5 \mathrm{~min}$ before being injected in LC (liquid chromatography). B-Butylation method: $50 \mu \mathrm{L}$ of diluted sample are mixed with $50 \mu \mathrm{L}$ of IS solution and are evaporated. After evaporation, $300 \mu \mathrm{L}$ of butanol / HCl 3mol/L are added and put in a water bath at $65^{\circ} \mathrm{C}$. for $15 \mathrm{~min}$. After a second evaporation step, the dry extracts obtained are taken up with $200 \mu \mathrm{l}$ of a $0.1 \%$ of a water solution $(0.01 \% \mathrm{FA})$.

Figure 4: SKML QCs analysis. The coefficients of variation for the two levels of CQs allow to evaluate the long term reproducibility for GAA (A) and creatine (B)

Figure 5 : Comparison of the two methods. A : Box plot of concentration of GAA and creatine obtained by IP method and by old method. A student test shows no significant difference. B : BLAND-ALTMAN graphs. The percentage of difference are within the range given by the approval limits $d \pm 2$ sdd. C : Passing Bablock regressions. They show strong correlations between the results of the two methods for the two molecules (GAA $r=0.991$, creatine $r=0.99$ )

Highlights

- Cerebral Creatine deficiency syndromes (CCDS)are responsible of non-specific neurological impairments with mental retardation

- LC-MS/MS measurements of guanidinoacetic acid (GAA) and creatine in urine and plasma are an important screening test to identify the deficit

- lon-pared method allows allow a greater retention of polar and basic molecules not retained on standard column and a simplicifaction of the technique. 

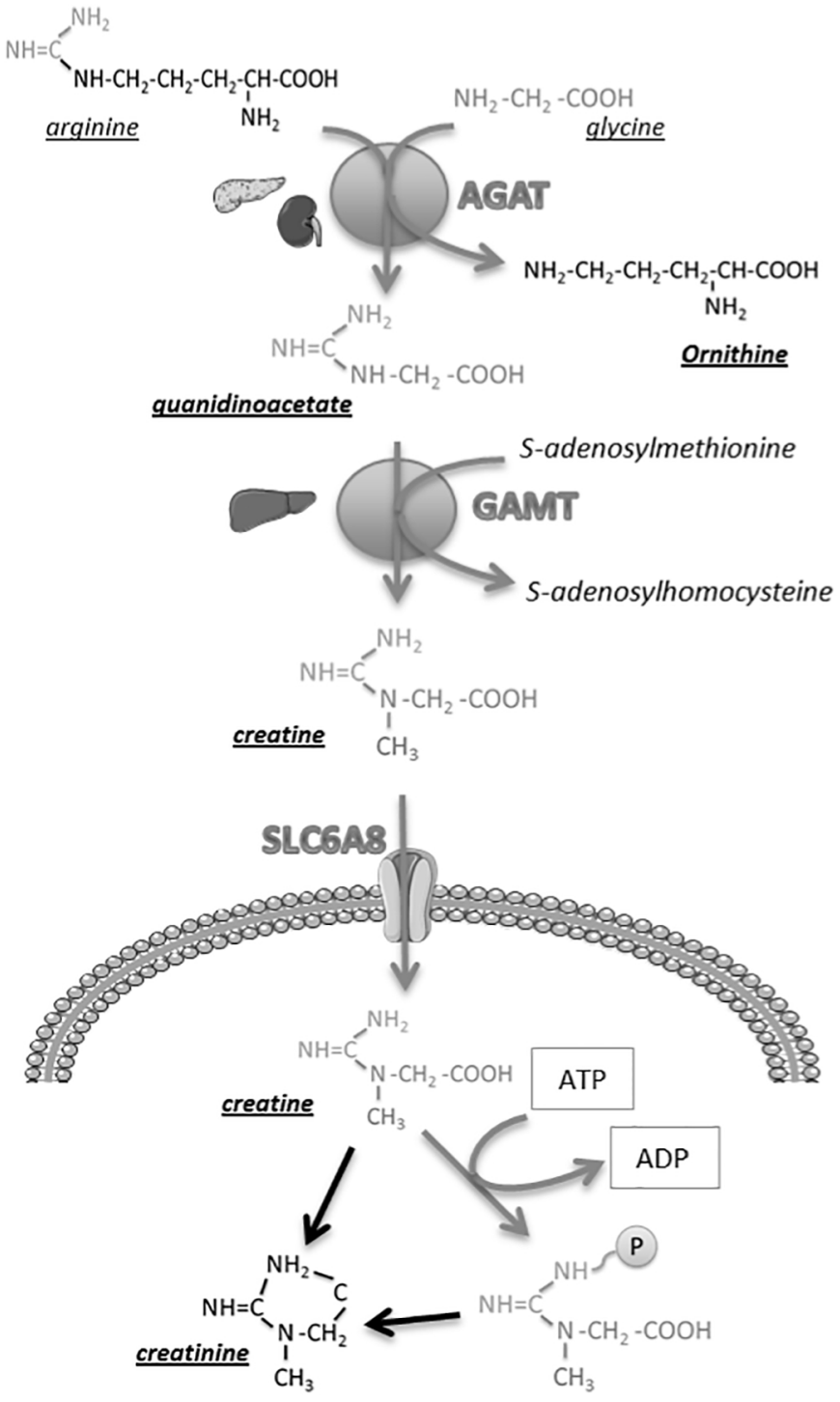

Figure 1 

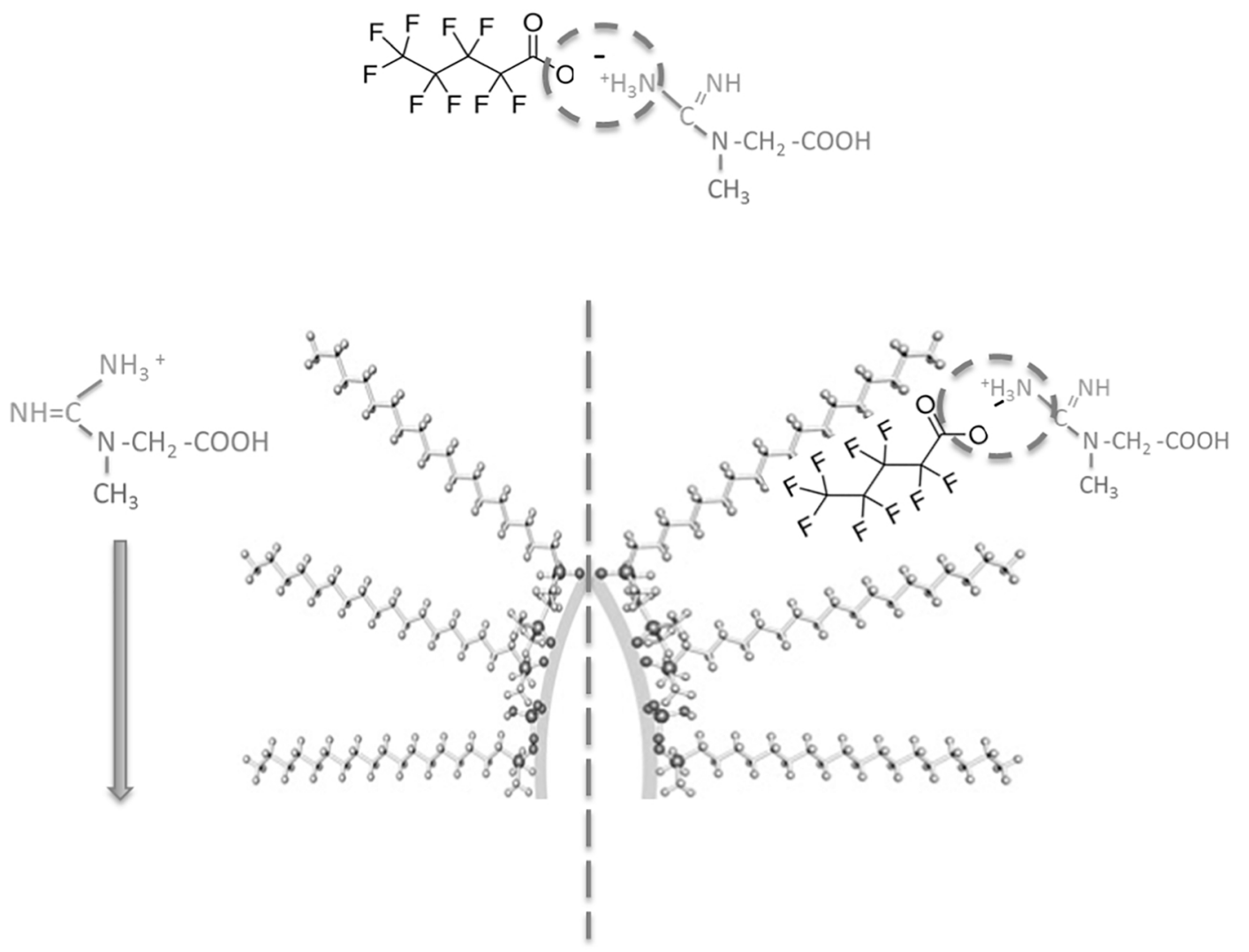

Figure 2 


\section{lon-pairing method}

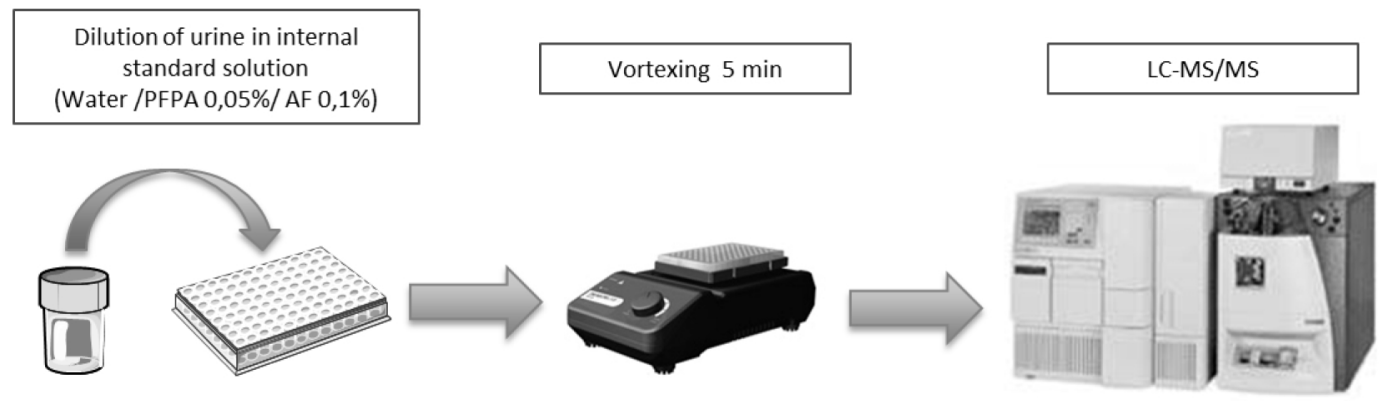

A

Butylation method:

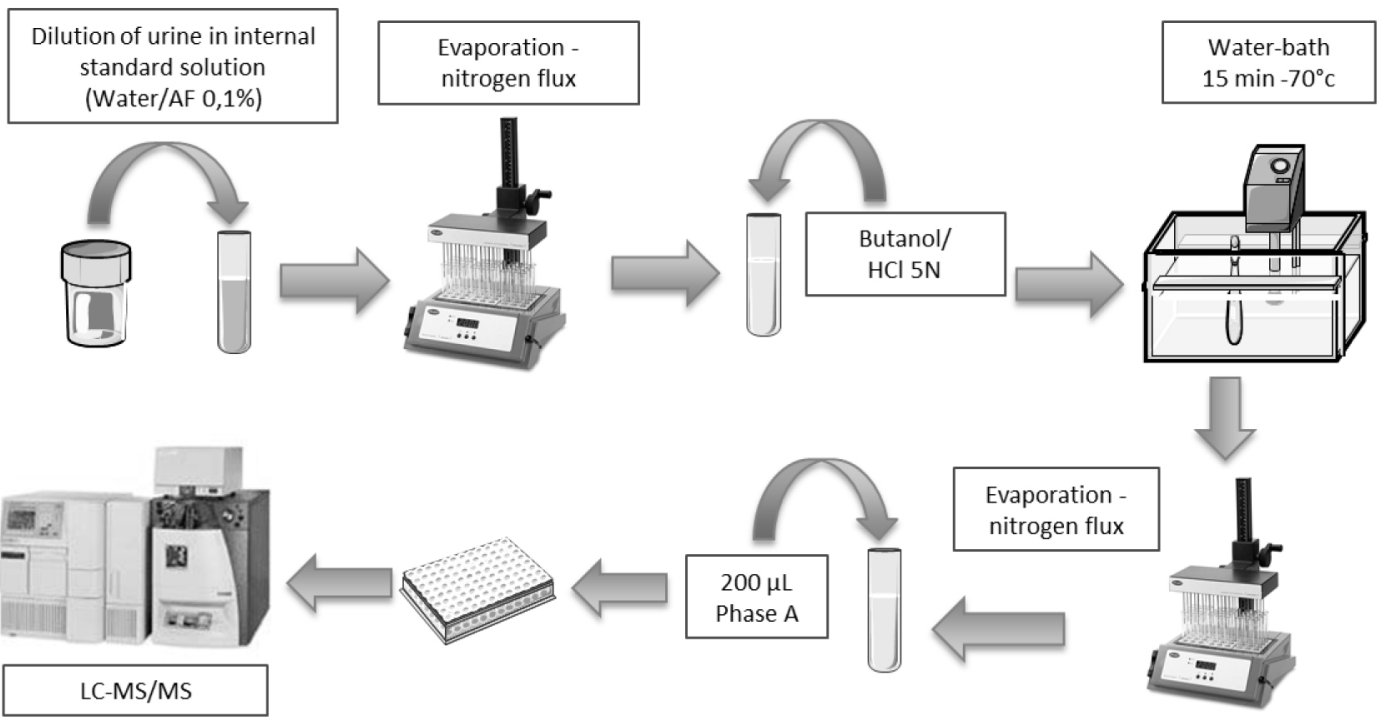

B 

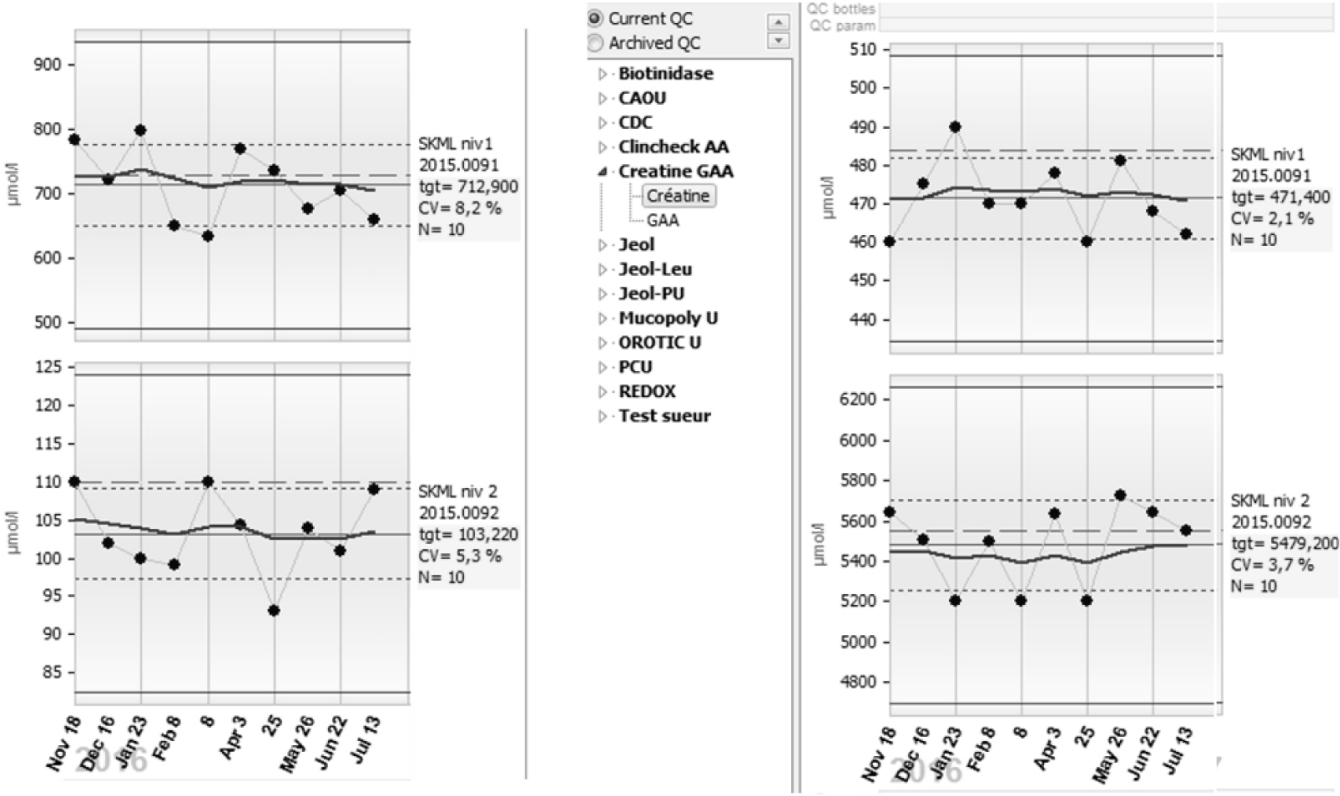

Figure 4 
A

\section{Creatine}

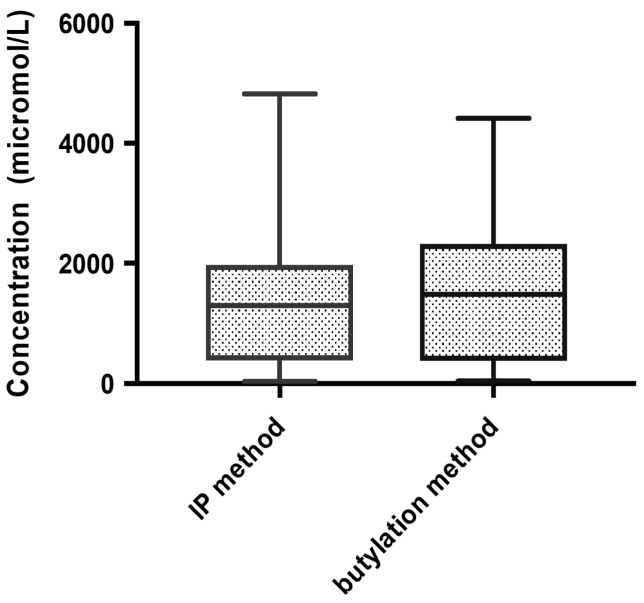

Creatine : butylation method - IP method
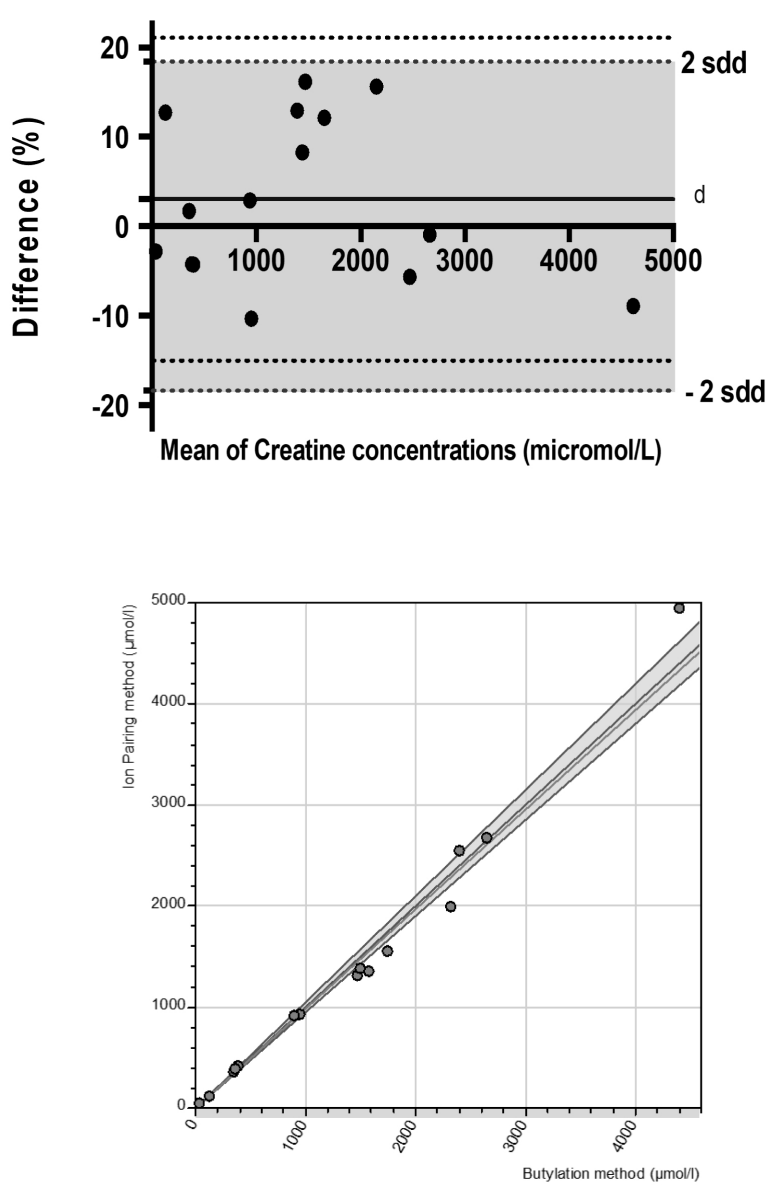

GAA

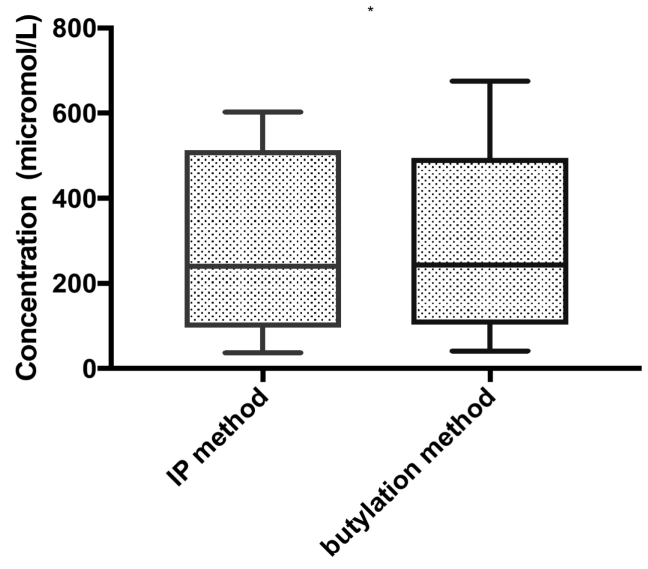

B
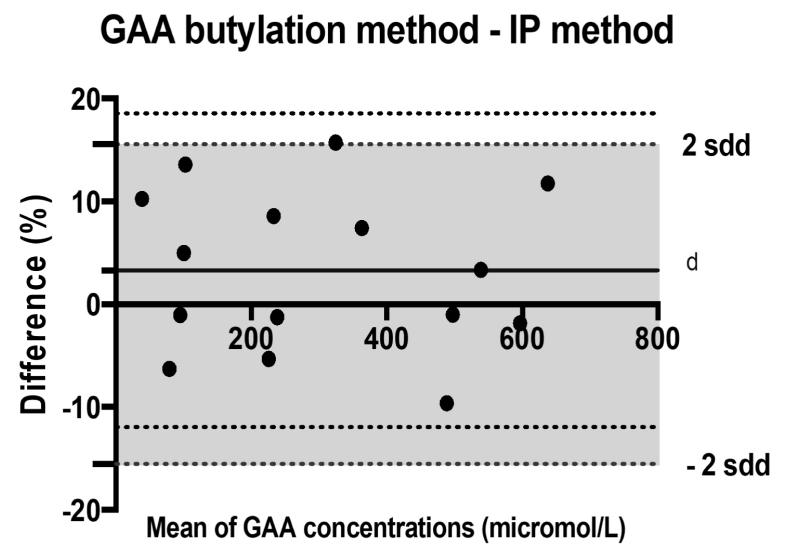

C

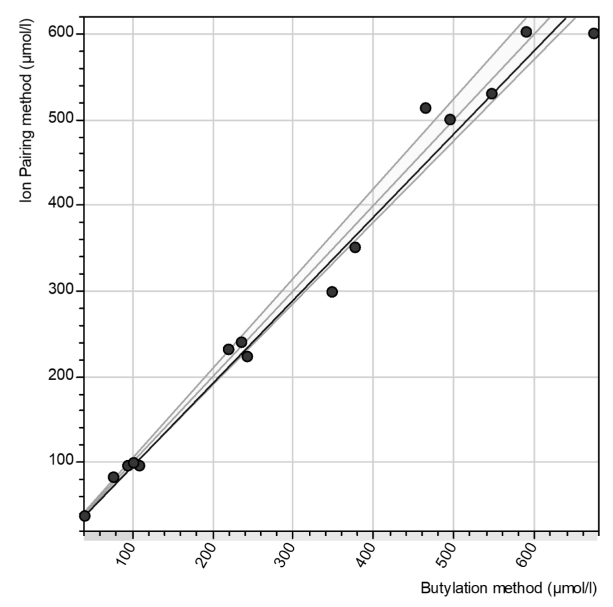

Figure 5 